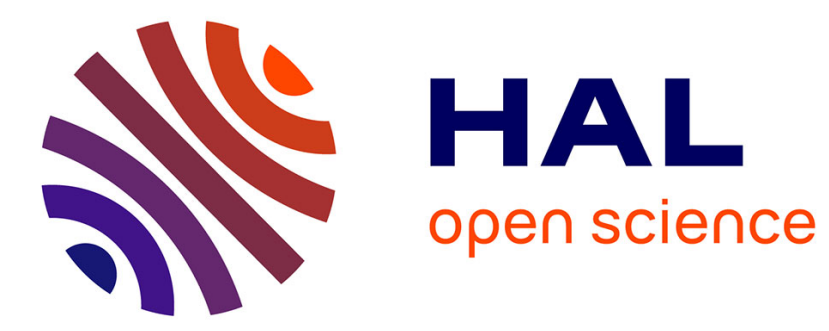

\title{
Une politique familiale à petits pas Normalisation de la garde partagée au Québec
}

Denyse Côté, Florina Gaborean

\section{To cite this version:}

Denyse Côté, Florina Gaborean. Une politique familiale à petits pas Normalisation de la garde partagée au Québec. Revue des politiques sociales et familiales, 2018. hal-02178398

\section{HAL Id: hal-02178398 \\ https://hal.science/hal-02178398}

Submitted on 9 Jul 2019

HAL is a multi-disciplinary open access archive for the deposit and dissemination of scientific research documents, whether they are published or not. The documents may come from teaching and research institutions in France or abroad, or from public or private research centers.
L'archive ouverte pluridisciplinaire HAL, est destinée au dépôt et à la diffusion de documents scientifiques de niveau recherche, publiés ou non, émanant des établissements d'enseignement et de recherche français ou étrangers, des laboratoires publics ou privés. 


\section{Une politique familiale à petits pas} Normalisation de la garde partagée au Québec

\author{
Mots-clés \\ - Québec \\ - Garde physique \\ partagee \\ - Politique familiale \\ - Garde parentale \\ - Séparation
}

\author{
Denyse Côté \\ Florina Gaborean \\ Sociologue - Université du Québec en Outaouais. \\ Anthropologue - Université d'Ottawa.
}

Au Québec, il y a trente ans, la garde physique partagée ${ }^{(1)}$ faisait figure de pratique contre-culturelle. Elle s'est depuis transformée en modèle : selon un sondage de 2013, 82 \% des Québécois estimaient que les enfants âgés de 6 à 12 ans devraient vivre en garde partagée suite à une rupture conjugale $^{(2)}$ alors que $23,2 \%$ des enfants de parents séparés vivaient effectivement en garde physique partagée

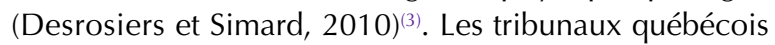
ont octroyé environ $21 \%$ de gardes partagées en 2008 et 2009 (Québec, 2011 b). Ceci est sans compter les nombreuses ententes hors Cour entre les parents séparés qui représentaient déjà, il y a dix ans, 63,1 \% des ententes de garde (Cyr et al., 2011; Juby et al., 2005 ; Canada, 1999)(4).

La préférence des tribunaux pour la garde partagée n'a pas fait l'objet, au Québec, d'un débat public ou de dispositions législatives comme ce fut le cas en France ou en Belgique (Côté et Gaborean, 2015). Cette modification de la politique familiale n'a pas été générée par le législateur (top down) et n'est pas survenue à la suite d'un débat public (bottom up), la rue ne s'étant pas mobilisée en faveur d'une telle mutation des normes. La garde physique partagée a plutôt reçu ses lettres de noblesse grâce à la convergence des pratiques parentales auxquelles se sont greffées certaines modifications administratives, judiciaires et professionnelles. De nouvelles normes postulant une égalité conçue en termes de présence symétrique de l'enfant auprès de chaque parent ont ainsi été consacrées. De même, l'élaboration d'un « nouvel ordre parental » hétérosexuel mettant l'accent sur la coparentalité et le maintien des rapports parents-enfants dans des situations de rupture familiale institue de nouvelles formes de régulation dans le champ des relations familiales. De nouveaux dispositifs d'intervention ont donc été mis en place pour soutenir l'exercice de la parentalité. Les intervenants du champ psychosocial ont incorporé ces nouvelles normes relatives à la parentalité et en ont fait leur promotion (Bastard, 2006). Le modèle du "bon divorce » et des normes exigeantes et uniformes relatives à la parentalité ont également été introduites en matière de régulation familiale (ibid., p. 16).

S'appuyant sur cette thèse, cet article s'intéresse aux évolutions opérées dans l'exercice des rôles parentaux après une rupture conjugale, en l'occurrence, aux modalités de mise en œuvre de la garde parentale, adoptées par les parents eux-mêmes ou promues par les instances institutionnelles qui participent à la prise en charge des enfants. La mise en place d'une préférence implicite pour la garde physique partagée au Québec consolide ainsi un modèle de garde parentale postdivorce selon des balises particulières relatives à l'intérêt de l'enfant. Ceci a été favorisé par des interventions opérées dans les champs juridique, administratif et psychosocial, guidées par une représentation de la garde physique partagée comme véhicule idéal de l'égalité. À cet effet, elles agiraient désormais, selon Benoît Bastard (2006), en tant que " police de la parentalité ». En même temps, ce modèle « idéal » de garde parentale postséparation est lui-même

\footnotetext{
(1) Pour des raisons de concordance culturelle et juridique, le choix a été de conserver l'expression " garde physique partagée » qui désigne, au Québec, le phénomène connu, en France, sous le vocable "résidence alternée ». L'expression " garde physique partagée " est définie au Canada et au Québec comme étant une entente selon les termes de laquelle les enfants passent au moins 40 \% de leur temps à la résidence de chaque parent (Côté et Gaborean, 2015).

(2) Le sondage rapporté par ces auteurs a été réalisé par Léger Marketing du 26 février au 11 mars 2013 auprès d'un échantillon de mille deux cent deux Québécois âgés de 18 ans et plus.

(3) Ce pourcentage varie selon l'âge de l'enfant et la période considérée. Il y aurait eu une augmentation progressive des gardes physiques partagées entre 1998 et 2002 pour les enfants de 4 ans (passant de $0 \%$ à 44,4\%), une diminution entre 2002 et 2004 pour les enfants entre 4 et 6 ans (passant de $44,4 \%$ à $21,1 \%$ ) et une augmentation pour les parents séparés depuis 2006 dont les enfants ont 8 ans et plus (33,6 \%) (Cyr et al., 2011, p. 28). Les ententes de garde partagée sont donc souples et se transforment au fil du temps.

(4) Seuls les cas de garde parentale ayant fait l'objet d'une ordonnance du tribunal sont recensés par Statistique Canada. Ils sont moins nombreux au Québec que les cas issus d'ententes à l'amiable entre parents, ceux-ci échappant, en fait, à l'œil du statisticien. Certains parents font entériner leur entente à l'amiable par un tribunal, mais ce sont plutôt les cas litigieux de garde parentale qui s'y retrouvent, ceux-ci représentant 10 \% de l'ensemble des cas. Selon Francine Cyr et al. (2011, p. 30), la garde physique partagée serait retenue par les parents dans 34,3\% des cas lorsqu'il s'agit d'une décision négociée entre eux.
} 
traversé par de nombreuses contradictions qui viennent parfois à l'encontre de la volonté des parents et des professionnels.

Cet article puise ses sources dans les dispositions du Code civil québécois et les politiques familiales, dispositifs $d^{\prime}$ intervention publique (dont des directives fiscales et administratives et les modalités d'octroi de la garde parentale - jurisprudence, médiation familiale) ainsi que dans les recherches sur les pratiques juridiques récentes. L'analyse s'appuie également sur des entretiens menés récemment auprès de trente-deux juges et avocats, médiateurs et parents en garde physique partagée. Enfin, les données empiriques ont été jumelées à celles de recherches antérieures, ce qui a permis de dresser un portrait des pratiques institutionnelles, professionnelles et parentales en matière de garde partagée au Québec.

Les dispositifs d'intervention familiale analysés permettent de saisir la double construction du modèle québécois de garde partagée : par l'offre, d'une part, c'est-à-dire à travers des pratiques institutionnelles et professionnelles incorporant les nouvelles normes familiales et sociales et, d'autre part, par les « bénéficiaires », les parents eux-mêmes, qui acceptent cette forme de régulation ou qui, au contraire, la rejettent, lorsqu'elle ne respecte pas leur volonté ou leur intérêt. L'enquête montre que cette régulation impérative de la parentalité ne prend pas toujours en considération la diversité des situations familiales et des manières de fonctionner après rupture. Certaines sont en effet évacuées ou normalisées, au nom d'une vision particulière de l'intérêt de l'enfant. Face à ces contraintes, certains parents et professionnels développent des stratégies complexes pour faire respecter leur vision et leurs désirs.

\section{Politiques familiales québécoises et nouvelles normes sociales}

Les politiques sociales contemporaines se sont adaptées aux multiples et profondes modifications morphologiques et socioanthropologiques des familles. Une privatisation des liens familiaux (Commaille, 2006) s'est ainsi installée, amenant les hommes et les femmes de familles hétérosexuelles à organiser leurs vies conjugale et familiale en fonction d'aspirations individuelles plutôt que de coutumes ou de normes exogènes. Dans ce contexte, les interventions judiciaires et sociales servent de plus en plus d'accompagnement aux décisions des acteurs concernés. La logique verticale de régulation des conflits, associée à la modernité (Wynants et al., 2009), se voit remplacée par une logique horizontale privilégiant la discussion et la négociation. À la norme imposée et contraignante se substitue le travail des acteurs en matière de coconstruction normative, et ce dans le cadre d'une transformation globale des dispositifs de régulation sociale et des rapports entre l'État et la société civile (Wynants et al., 2009). La frontière entre la sphère privée et l'ordre politique s'en trouve ainsi fragilisée : des acteurs plus nombreux participent à la production et à la mise en œuvre de politiques sociales et agissent beaucoup plus souvent dans l'ombre (Commaille, 2006).

Dans le cas de la garde partagée, les nouvelles normes $s^{\prime}$ inspirent particulièrement de demandes sociales en matière d'égalité hommes-femmes et de démocratisation de la famille, ce que résume ainsi ce répondant:

- "Je pense que c'est le rapport hommes-femmes qui a changé, la société et les demandes de la société aussi. Les rôles des hommes et des femmes ont changé également, ce qui fait en sorte que la loi change, la loi s'adapte aux gens, aux besoins, aux mours » (avocatmédiateur).

Au Québec, la nouvelle régulation sociale des liens familiaux prend racine dans les transformations culturelles et politiques issues de la modernisation des rapports sociaux émanant de la Révolution tranquille des années 1960. L'idéal d'égalité hommes-femmes porté à l'origine par un fort mouvement social s'est transformé au tournant du siècle en fondement idéologique des politiques sociales et familiales. Tant et si bien que le modèle québécois semble désormais fondé sur une conception symétrique des rôles parentaux et sur la nature contractuelle des rapports conjugaux, présentés comme le fondement de l'égalité des droits des femmes et des hommes. Dans ce contexte, la garde partagée incarne les valeurs émergentes du couple hétérosexuel (Côté, 2004) en matière de démocratie et de mobilité conjugale, la liberté de chaque homme ou femme de choisir son mode de vie conjugal (Giddens, 2004) et parental. Elle répond aussi aux besoins des parents à double insertion professionnelle, dans une société où l'économie domestique a été largement remplacée par un marché des biens et services destinés aux familles (garderies, services associatifs, publics ou privés conçus spécifiquement pour les enfants). La symétrisation des liens et des rôles parentaux que suppose la garde partagée correspond aussi au concept contemporain de "I'individu-citoyen » (homme, femme ou enfant) débarrassé des contraintes ataviques, sexistes et âgistes de la famille 
patriarcale. Ceci conduit certains à considérer la garde physique partagée comme garante de l'égalité entre les pères et les mères.

La garde partagée répond à l'idée que les enfants, en cas de séparation parentale, auraient droit à un accès symétrique (en temps de présence) à chaque parent, et que ce contact symétrique serait un droit des pères. La symétrie des rôles et du temps de présence parentale qu'offre la garde partagée reflète ainsi concrètement l'idée d'une autre norme récente, celle de la pérennité du lien parental (Côté et Gaborean, 2015). Parallèlement, apparaît au Québec une confusion entre le principe d'autorité parentale conjointe consacré par le Code civil québécois et la pratique de la garde physique partagée (Tétrault, 2014). Ces phénomènes émergent au moment où l'expertise parentale est de moins en moins reconnue par les tribunaux et se trouve remplacée par des expertises professionnelles diverses (médiateurs, psychologues, psychiatres, travailleurs sociaux).

Ces mutations se reflètent dans les politiques familiales encourageant le partage des tâches entre les conjoints (Québec, 1997 et 2011 a,) et dans les mesures d'appui à la double insertion professionnelle visant à promouvoir l'égalité des sexes. Grâce à ces mesures, graduellement mises en place à partir des années 1990, le Québec s'est doté d'une politique familiale considérée comme l'une des plus généreuses au monde. Ainsi, un programme universel de services éducatifs à l'enfance adopté en 1997 permet aux familles d'avoir accès à un service de garde à prix modique. En outre, le régime québécois d'assurance parentale instauré en 2006 offre des congés généreux et flexibles autant aux mères qu'aux pères. Piliers de la politique familiale québécoise, ces programmes favorisent I'autonomie financière des mères et la prise en charge plus régulière par les pères du soin de leurs enfants. Non sans succès : I'activité professionnelle des mères s'est accrue considérablement ${ }^{(5)}$ et les pères se prévalent de plus en plus du congé de paternité(6). Une norme gouvernementale unique au monde encourage aussi les milieux de travail à intégrer la conciliation travail-famille dans la gestion de leurs ressources humaines (Québec, 2010). Spécifiant les exigences en cette matière et s'adressant aux organisations publiques et privées, cette norme est assortie d'un programme de certification. Depuis son émergence, la conciliation famille-travail se retrouve au cœur de la politique familiale, notamment de la politique en matière d'égalité femmes-hommes (Québec, 2011 a), et des plans d'action qui en ont découlé(7).

\section{Modifications en matière de garde parentale}

Le cadre institutionnel a évolué au Québec vers une préférence implicite pour la garde physique partagée sans que le Code civil ait été pour autant modifié. Pour saisir comment s'est opéré ce changement, il est nécessaire de comprendre à la fois le contexte juridique mais aussi les dispositions administratives mises en œuvre, ainsi que l'évolution des dispositifs professionnels. Ces éléments seront mis en rapport avec les expériences des juges, des avocats-médiateurs et des parents interviewés.

\section{Contexte juridique}

Au Québec, le gouvernement fédéral (canadien) et la province du Québec se partagent la compétence juridique en matière de garde parentale : le Code civil québécois (1991) et la Loi canadienne sur le divorce (1985) s'appliquent ici. Le Code civil québécois a pleine juridiction en matière $d^{\prime}$ octroi de la garde parentale dans les cas des enfants nés hors mariage, qui représentent plus de 65 \% des naissances au Québec (Québec, 2010). La Loi canadienne sur le divorce ne s'applique qu'aux couples mariés qui font une demande de divorce, mais les dispositions québécoises sur les pensions alimentaires, la garde et le droit de visite s'appliquent aux couples mariés et à ceux en union de fait. Tous les parents québécois séparés ou divorcés conservent ainsi des droits et des devoirs envers leurs enfants après une séparation, indépendamment de leur statut marital ou du mode de garde parental retenu : les pressions du mouvement féministe ont permis d'inscrire l'autorité parentale conjointe au Code civil québécois dès 1981. Les débats publics qui ont mobilisé la France et la Belgique à ce sujet (Côté et Gaborean, 2015) sur la garde partagée n'ont pas eu lieu au Québec. Cela dit, les mêmes enjeux sont tout de même observés chez les psychologues, sans écho médiatique cependant. Ils se limitent plutôt à la pertinence de la garde partagée pour les enfants d'âge préscolaire et à l'importance d'un parent principal pour le développement d'un sain attachement chez l'enfant (Cyr, 2008 ; Gauthier, 2008 ; Cyr, 2006). On en retrouve aussi certaines traces au sein des tribunaux lorsque les experts juridiques ou

\footnotetext{
(5) En 2010, les femmes représentaient déjà 47,8 \% de la population active au Québec (Québec, 2011 a).

(6) Selon les données de l'Institut de la statistique de Québec (ISQ), 76 \% des pères ont pris leur congé de paternité en $2010-2011$ (qui a été mis en place en 2006) (Québec, 2011 c).

(7) La mise en œuvre du plan d'action 2011-2015 est estimée à 290 M\$ (Québec, 2011 a).
}

Revue des politiques sociales et familiales $n^{\circ} 128-3^{\text {e }}$ trimestre 2018 
psychosociaux présentent leurs positions, souvent contraires, à ce sujet. Ces avis sont souvent établis à partir de valeurs et de croyances personnelles des différents acteurs de la scène juridique et assistancielle, ce qui aurait mené à un usage biaisé des données empiriques disponibles (Cyr, 2008) $)^{(8)}$.

Au Québec, même si la garde est confiée plus fréquemment à la mère ${ }^{(9)}$, il s'est tout de même installé, à la Chambre de la famille, une préférence implicite pour la garde physique partagée, c'est-à-dire une tendance des juges à accorder la garde partagée sans que des directives à cet effet n'aient été formellement consignées (Gagnon, 2006). Les jugements issus de ce tribunal sont marqués par cette forte tendance à privilégier la garde partagée (Tétrault, 2014) et ceci constituerait, selon certains, une présomption de facto (Tétrault, 2014 ; Gauthier, 2008 ; Le Roy, 2006 ; Joyal, 2003). Elle résulte entre autres de la demande croissante d'une garde partagée par des pères davantage impliqués auprès de leurs enfants, mais surtout de l'application d'une conception du principe juridique émis par les autorités canadiennes selon lequel il serait dans l'intérêt de l'enfant de bénéficier du maximum des contacts avec chacun de ses parents : «.... le principe de maximisation est poussé dans ses derniers retranchements pour expliquer l'établissement d'une garde partagée » (Tétrault, 2014). Ce principe juridique de l'intérêt de l'enfant est depuis longtemps au cœur des décisions de la Chambre de la famille québécois en matière de garde parentale, mais reste flou et changeant. En raison de son caractère subjectif, il reflète souvent les croyances et les expériences personnelles des professionnels en droit familial ainsi que les valeurs sociales du moment (Goubau, 2009).

La tendance du tribunal à octroyer la garde partagée est si forte (ibid.), contrairement à ce qui se passe en France (Neyrand, 2014), que la Cour d'appel ${ }^{(10)}$ a dû rappeler formellement aux acteurs juridiques l'absence d'une présomption de garde physique partagée au Québec (Tétrault, 2014) ${ }^{(11)}$. Cependant, la garde partagée y est de plus en plus privilégiée par les juges, même en situation de désaccord important entre les parents (Tétrault, 2014 ; Le Roy, 2006 ; Joyal, 2003), de conflit parental soutenu ou de violence conjugale (Côté, 2012)(12). Pour certains experts, cette tendance est en voie de diminution; pour d'autres, au contraire, elle s'est consolidée, ce que semblent confirmer les quelques recherches disponibles sur le sujet. Dans les jugements de gardes partagées litigieuses entre 2003 et 2006 qu'elle a étudiés, Inès Le Roy (2006) relève $70 \%$ de jugements ayant octroyé une garde partagée. Près des trois quarts (sept sur dix) des juges de la Cour supérieure du Québec interviewés par Renée Joyal (2003, p. 274) et siégeant régulièrement à la Chambre de la famille de Montréal ont affirmé privilégier clairement la garde partagée, ce que confirment les perceptions d'experts, de juristes, d'observateurs et d'intervenants québécois. L'argument de ces juges est simple : dans l'intérêt de l'enfant, il faudrait "minimiser les effets du divorce sur les liens parent-enfant, sur les liens père-enfant notamment, souvent affaiblis à la suite d'une rupture conjugale. I/s pensent ainsi préserver l'unicité de la famille d'origine et "la continuité de son histoire familiale» » (ibid.). Confirmant ces analyses, les avocats québécois en droit de la famille ainsi que les juges interrogés dix ans plus tard affirment qu'ils n'ont plus à démontrer au tribunal le bien-fondé d'une demande de garde partagée, qu'ils doivent, au contraire, "établir les motifs [de] pourquoi on n'en veut pas » (Joyal, 2004, p. 453). La garde partagée serait souvent demandée par les pères et de plus en plus accordée par les juges.

- "On sent vraiment un mouvement de la part des tribunaux de favoriser la garde partagée, beaucoup plus $q u$ avant » (avocate-médiatrice).

- "On en est rendu là qu'il faut vraiment trouver une raison très solide pour qu'il n'y ait pas de garde partagée...» (avocat-médiateur).

- "La garde partagée est entrée dans les mentalités au point où certains juges à certains moments, je dirais surtout à partir des années 2000, ont commencé à se faire reprocher d'y être trop favorables... Donc il ne

\footnotetext{
(8) À cet effet, une étude québécoise de 2012, faisant d'ailleurs écho à de nombreuses études américaines et européennes, compare la garde monoparentale à la garde partagée sous I'angle de leurs impacts sur l'adaptation de l'enfant. Les résultats montrent que les deux modalités de garde sont équivalentes. Selon la même étude, les modalités de garde ne jouent pas un rôle modérateur en cas de conflit parental. L'étude réhabilite en quelque sorte la garde unique qui, selon I'auteur, peut être affectée négativement par des préjugés sociaux importants (Ouellet, 2012, p. 52).

(9) Selon I'ISQ, 65,9 \% des gardes sont confiées à la mère lors de la séparation des parents. L'arrangement de garde le plus fréquent est celui où l'enfant vit avec sa mère et voit son père régulièrement (41\%) (Desrosiers et Simard, 2010).

(10) Les critères retenus par la Cour d'appel relatifs à l'attribution d'une garde partagée sont multiples : l'intérêt de l'enfant, la stabilité, la capacité parentale comparable, la capacité des parents de communiquer entre eux sur les questions qui concernent l'enfant, la proximité des résidences parentales, l'absence de conflits entre les parents et le désir de l'enfant lorsqu'il est suffisamment vieux (Tétrault, 2014, p. 13).

(11) Entretiens avec des juges québécois en matière familiale.

(12) Ceci est confirmé par des intervenantes en maison d'hébergement interviewées récemment.
}

Revue des politiques sociales et familiales $\mid n^{\circ} 128$ - $3^{\text {e }}$ trimestre 2018

38 Politique familiale 
s'agit pas de les convaincre que ça peut être une bonne formule de garde ou que ce n'est pas une formule qui est contre l'intérêt de l'enfant» (juge).

La garde partagée serait même octroyée dans des contextes de conflit important et durable entre les parents, de violence postséparation ou même, lorsqu'il y a soupçons d'abus sexuel.

- "Une de mes clientes soupçonnait que l'enfant avait été abusé sexuellement et ça a été compliqué, parce $q u ' i l$ y avait plusieurs niveaux, plusieurs espaces qui étaient impliqués. Ils ont à ce moment-là imposé, avec les recommandations de la travailleuse sociale, une garde partagée de façon progressive. Je n'ai jamais vu dans ma pratique quelque chose de si drastique » (avocat-médiateur).

Selon plusieurs avocats, en l'absence de balises claires guidant l'application du critère du « meilleur intérêt de l'enfant » qui, en l'occurrence, reste toujours flou, les jugements de garde "à la Salomon » se multiplieraient : il s'agit de jugements octroyant la garde partagée sans prise en compte réelle des capacités de chaque parent. L'octroi de la garde partagée ne se ferait pas alors véritablement au cas par cas, mais plutôt selon les préférences de certains juges pour la garde partagée car ils disposent à cet effet d'un important pouvoir discrétionnaire :

- "Les juges arrivent avec une idée préconçue que les parents sont entre guillemets "égaux»... Ce n'est plus [...] du cas par cas. On devrait se pencher sur [...] l'intérêt de l'enfant... » (avocate-médiatrice).

Or, l'imposition d'une garde physique partagée aux parents ne donne pas de bons résultats. Ils ne renoncent pas nécessairement à leur demande initiale de garde paternelle ou maternelle, et les procédures judiciaires s'étirent. Selon des avocats interrogés, il serait plus judicieux pour la Chambre de la famille de considérer I'option de la garde partagée avec prudence dans les cas litigieux :

- "Les gardes partagées imposées, ce n'est pas des bons succès. Parce qu'il y a un parent qui est malheureux en partant d'avoir perdu sa garde. Quand tu dis «une garde partagée imposée», ça tiraille, puis ça continue de tirailler. Alors c'est difficile pour un parent malheureux qui ne fait pas une entente de garde partagée de vivre la garde partagée. Ça, là, je m'en rends compte moi dans ma pratique » (avocate-médiatrice).
De plus, le partage symétrique du temps de garde n'est pas toujours réaliste, en particulier dans les cas d'enfants très jeunes ou de bébés allaités.

- "Je pense qu'il y a beaucoup de droits personnels là-dedans. C'est une question de droits, les pères ont les mêmes droits que la mère, donc pourquoi qu'ils se contenteraient de moins que cinquante-cinquante?" (avocate-médiatrice).

Le recours au droit collaboratif et au principe de justice participative est de plus en plus fréquent : les parents font moins appel aux services d'un avocat lorsqu'ils se présentent au tribunal et acceptent plus facilement de conclure une entente permettant de limiter le coût des procédures judiciaires. "Faire des compromis » extrajudiciaires est devenu l'équivalent, dans l'opinion publique québécoise, $d^{\prime}$ « avoir appris à divorcer » ou d'un «bon divorce ». De là il n'y a qu'un pas pour associer le choix de la garde partagée à la capacité pour les parents de " faire des compromis".

- "Au début de la garde partagée, on se disait que cela va être l'exception, puis maintenant c'est devenu carrément la norme... La nouvelle génération [est] plus [prête] à faire la garde partagée que l'ancienne génération... Ils travaillent [...] à synchroniser leur calendrier de travail pour partager la responsabilité par rapport à l'enfant » (avocat-médiateur).

S'opère ici un glissement de valeurs : l'idéal de l'égalité des droits se voit traduit désormais en symétrie du partage des tâches parentales (Hachet, 2014).

\section{Directives fiscales et administratives en matière de garde partagée}

Certaines nouvelles directives gouvernementales reflètent la normalisation de la garde partagée au Québec. Ainsi, un nouveau régime de fixation de pensions alimentaires, entré en vigueur en 1997, a facilité I'exercice de la garde partagée en incluant le temps de garde parental dans le calcul des pensions alimentaires prélevées à la source (Québec, 2014 a). Reprenant à son compte des directives du gouvernement canadien, les règles québécoises considèrent depuis lors qu'il y a garde partagée lorsqu'un enfant réside $40 \%$ du temps dans deux résidences parentales distinctes (Canada, 1997). La pension alimentaire est donc fixée si un écart important est constaté entre les revenus des parents, et ce, afin de répondre aux besoins des enfants de la même façon qu'avant la séparation parentale. Selon 
les avocats interrogés, ces modalités de calcul augmentent cependant le quotient de difficulté des règlements à I'amiable.

- "À cause des pensions imposées de part et d'autre, le parent qui aurait à payer la pension alimentaire se bat beaucoup plus pour la garde partagée afin de réduire son obligation alimentaire » (avocate-médiatrice).

Par ailleurs, depuis 2011, la Loi canadienne de l'impôt sur le revenu donne accès à parts égales aux parents en garde partagée aux déductions fiscales ainsi qu'à la Prestation fiscale canadienne pour enfants (Canada, 2017). Au Québec, le Soutien aux enfants ${ }^{(13)}$ est désormais versé simultanément aux deux parents en garde partagée, tout comme le supplément pour enfant handicapé (Québec, 2014 b). Ces directives administratives québécoises et canadiennes facilitent l'exercice de la garde partagée car elles permettent d'alléger les négociations financières entre les parents et diminuent, du coup, les tensions liées à celles-ci.

\section{La médiation familiale : nouvel outil de gestion de la garde parentale} Le législateur québécois a également mis en place, en 1997, un nouveau système de gestion des ruptures conjugales axé sur la médiation et destiné aux couples avec enfant (Québec, 2013)(14). Clairement ancrée dans un paradigme de justice collaborative, cette mesure a été centrale à la normalisation du concept de symétrie parentale au Québec et, in extenso, de la garde physique partagée. Dans les lignes qui suivent, son articulation est présentée afin d'illustrer l'introduction de cette nouvelle norme de garde parentale par le biais d'un encadrement à la fois administratif, professionnel et juridique.

Le souci de faciliter le processus de séparation, d'instaurer la communication entre les parents, de limiter les coûts de la séparation, de rendre cette séparation plus accessible et de désengorger les tribunaux ont motivé cette réforme (Québec, 1997 ; Belleau et Talbot-Lachance, 2008). Le nombre de séparations conjugales étant en constante augmentation, pour le législateur, il s'agissait de contrer certains effets pervers du système conflictuel propre à la Chambre de la famille. Selon les concepteurs de cette politique publique, la médiation familiale devait également permettre de redonner aux parents en voie de séparation un contrôle sur le processus. Quelques années plus tard, le ministère de la Justice soulignait d'ailleurs que $82 \%$ des parents ayant fait appel au Service de médiation de la Chambre de la famille seraient parvenus à une entente et auraient déboursé deux fois moins que les parents ayant opté pour la Chambre de la famille (Québec, 2012 a) ${ }^{(15)}$.

Le gouvernement du Québec a investi de façon importante dans ces services de médiation familiale et a établi certaines contraintes : les parents voulant faire appel au tribunal se voient désormais obligés de participer à une séance obligatoire d'information sur la médiation familiale avant de pouvoir y avoir accès. Cette séance a été remplacée en 2013 par une séance obligatoire sur la coparentalité après rupture. Celle-ci reflète la nouvelle philosophie de la jurisprudence récente : I'enfant aurait droit à un accès symétrique à ses parents suite à la séparation conjugale. Cette séance fait donc désormais la promotion de la médiation et de la résolution extrajudiciaire des conflits parentaux. Six rencontres de médiation gratuites sont même offertes aux parents ${ }^{(16)}$ et cinq ordres professionnels ont été appelés à accréditer des médiateurs familiaux ${ }^{(17)}$. Les médiateurs et médiatrices rencontrés dans la région de l'Outaouais ${ }^{(18)}$ sont aussi accrédités par le Barreau du Québec. Ils se voient comme des accompagnateurs de leurs clients dont les situations sont souvent complexes. Selon leurs dires, leurs outils d'intervention sont leur formation initiale en droit, leurs expériences professionnelles et les compétences développées par la suite en matière de médiation ${ }^{(19)}$. Leur travail se structure autour de solutions souvent déjà mises en œuvre par leurs

\footnotetext{
(13) Le « Soutien aux enfants » est une mesure fiscale destinée aux familles ayant des enfants à charge âgés de moins de 18 ans. Elle varie en fonction du revenu familial, de la situation conjugale des parents ainsi que du nombre d'enfants à charge.

(14) Ceci renvoie à la notion de «maximum de communication».

(15) À la suite d'une enquête menée auprès des personnes ayant assisté aux séances offertes dans le cadre du projet pilote, plus de $97,5 \%$ des répondants ont affirmé avoir apprécié cette formule et 89 \% ont indiqué que la séance les avait outillés pour régler leur séparation (Québec, 2012 a).

(16) Ces séances sont offertes dans quarante-deux palais de Justice du Québec.

(17) Il s'agit du Barreau du Québec, de la Chambre des notaires du Québec, de l'Ordre des conseillers et conseillères d'orientation et des psycho-éducateurs et psycho-éducatrices du Québec, de l'Ordre des psychologues du Québec et de l'Ordre professionnel des travailleurs sociaux du Québec. Les employé-e-s des centres jeunesse peuvent aussi être accrédités comme médiateurs.

(18) L'Outaouais est l'une des dix-sept régions situées à la frontière ouest du Québec.

(19) L'accréditation en médiation familiale comprend un cours de soixante heures, dix mandats de médiation sous supervision ainsi que quarante-cinq heures de formation supplémentaire. Six de ces cent cinq heures portent sur la violence conjugale. Aucune formation en sociologie de la famille ne fait partie de ce
} curriculum. 
clients, auxquelles s'ajoutent certaines propositions de leur cru.

- "La médiation, on est comme des éducateurs, on les informe, on les motive. On les amène à aller voir ou à réfléchir à d'autres pistes de solutions. Ils sauvent beaucoup de frais » (avocate-médiatrice).

Les séances de médiation dépasseraient cependant les aspects purement juridiques de la rupture pour inclure des aspects émotifs, relationnels et financiers : la médiation " humaniserait » ainsi, selon eux, la négociation entre les conjoints (Belleau et Talbot-Lachance, 2008). Ils doivent ainsi recourir à d'autres professionnels (coachs de vie, travailleurs sociaux, psychologues), les problèmes soulevés en médiation dépassant leurs propres compétences juridiques.

- «Ce dont les [parents] ont besoin, c'est du support. C'est pourquoi je travaille avec beaucoup de professionnels. Parce que, quand les clients soulèvent un besoin, je suis capable de les référer pour qu'ils reviennent » (avocat-médiateur).

Légitimée par une contrainte légale, accompagnée d'un discours sur les bienfaits de la séparation non conflictuelle et de la capacité de tout parent d'arriver à une entente consensuelle, la médiation familiale est donc soutenue par les médiateurs rencontrés qui la présentent à leurs clients comme la solution idéale aux conflits parentaux ${ }^{(20)}$. Ce discours est partagé par de nombreux professionnels, médiateurs accrédités, travailleurs sociaux et psychologues en exercice ainsi que par leurs corporations respectives. La médiation superpose à l'autorité arbitrale du tribunal un système professionnel dont l'objectif est de contraindre les parents à s'entendre lorsqu'ils n'auront pas réussi à le faire seuls (Guillemard, 2012). Comme le souligne B. Bastard (2012), elle se présente paradoxalement comme une intervention impartiale tout en faisant la promotion d'un mode spécifique de garde parentale, celui de la division symétrique du temps de présence des enfants à chaque domicile parental. La médiation occupe ainsi une place de choix à la Chambre de la famille du Québec. Il s'agit $d^{\prime}$ ailleurs du seul secteur où la médiation se substitue au dispositif juridique. À titre d'exemple, le législateur québécois a prévu, dans un autre cas, celui d'une autre cour, la Division des petites créances, la possibilité d'une médiation sans retirer pour autant la possibilité aux justiciables de déposer une plainte directement au tribunal. Dans le cas de la garde postséparation, les parents ne peuvent plus déposer leur dossier directement à la Chambre de la famille : ils doivent obligatoirement considérer la médiation familiale avant de pouvoir le faire. Or, si la finalité de la médiation familiale rejoint celle de la Chambre de la famille, à savoir le rétablissement d'une paix sociale et familiale, le retrait de ce droit aux couples parentaux vivant d'importants conflits ou de la violence suscite des interrogations (Guillemard, 2012).

Les parents québécois désirant se séparer ont en effet perdu leur accès direct au système judiciaire lorsque l'obligation de suivre des sessions sur la coparentalité après rupture offertes par le service de médiation de la Chambre de la famille a été décrétée. Ces sessions dirigent les parents vers un modèle particulier, celui d'une résolution de conflits en matière familiale où les parents devront avoir préalablement négocié par l'intermédiaire d'avocats ou d'un médiateur (Clairmont et al., 2014). Leurs avocats respectifs devront fournir une preuve écrite de leur présence à ces sessions avant qu'une date d'audition ne soit fixée. Or, ces sessions font la promotion d'une "démocratie » négociée entre les ex-conjoints, de la responsabilité individuelle des plaignant-e-s, de la médiation comme « impartiale » et de l'entente négociée en médiation comme nécessairement « équitable » (Clairmont et al., 2014). La médiation familiale se transformerait ainsi symboliquement en « démocratie de l'accompagnement familial » (Sassier, 2001) et articulerait une nouvelle norme, celle de l'autorégulation familiale, de l'autodétermination et I' « autonomie » des individus (Commaille, 2006) ${ }^{(21)}$. C'est en effet sous cet angle que les documents officiels présentent la séance obligatoire de médiation familiale.

- "Cette séance ${ }^{(22)}$ a pour objectif d'assurer à tous les parents la possibilité [...] de voir la médiation présentée

(20) En Outaouais, des médiateurs familiaux dont la profession initiale est le droit ont été interrogés, ce qui correspond au profil de la majorité des médiateurs accrédités de cette région.

(21) Cette tendance s'observe dans tous les pays occidentaux. Dans un article paru en 2014, Catherine Collombet souligne l'augmentation des pratiques de médiation en Europe, en particulier à la suite de l'adoption de la directive 2008/52/CE qui recommande aux États de l'Union européenne de prévoir la possibilité de recourir à la médiation dans les litiges familiaux et de donner un caractère exécutoire aux accords qui en sont issus. Cependant, dans la plupart des pays européens, la médiation demeure optionnelle pour les parents en voie de séparation. La Norvège est cependant le seul pays où la médiation est obligatoire avant saisie du tribunal pour les parents mariés ayant des enfants âgés de moins de 16 ans (Collombet, 2014, p. 76).

(22) Contenu de cette séance développée par le Comité des organismes accréditeurs en médiation familiale (COAMF) sur mandat du ministère de la Justice du Québec et « [respectant] l'obligation d'information prévue à l'article 814.3 Code de procédure civile [sic] » (Québec, 2012 b).

Revue des politiques sociales et familiales $n^{\circ} 128-3^{\text {e }}$ trimestre 2018

41 Politique familiale 
comme un modèle de résolution de conflit et un moyen de mettre en place un nouveau modèle pour exercer la parentalité après rupture »(Québec, 2012 b).

Cette séance d'information obligatoire ne présente pas la garde partagée comme un modèle supérieur de garde parentale. Mais celle-ci s'impose dans ce cadre à titre de prolongation naturelle de l'idéal coparental mis de I'avant dans cette séance et rejoint le précepte juridique canadien du "maximum de communications » entre parents séparés ainsi que le discours public sur la symétrie des rôles parentaux et le droit d'accès symétrique de I'enfant à ses deux parents. Certains formalisent même les rapports entre parents séparés sous forme d'une Charte de parentalité (Clairmont et al., 2013) ${ }^{(23)}$ ou d'un contrat de coparentalité (Juston et Gargoullaud, 2014) alors que, parallèlement, I'exception négociée au Québec en 1997 pour les femmes victimes de violence semble s'estomper. Le législateur avait en effet alors prévu au Code de procédure civile québécois un mécanisme pour les exempter de la séance obligatoire, les rapports violents étant peu propices à une négociation. Or, aucune mention de cette exemption ne figure dans la documentation disponible relative aux séances obligatoires sur la coparentalité. Des intervenantes en maison d'hébergement rapportent également que les démarches pour obtenir cette exemption, bien que toujours présentes dans les directives gouvernementales, sont maintenant de moins en moins accessibles. Les femmes victimes de violence se voient proposer la médiation comme solution à leur situation marquée, justement, par la violence, malgré les recherches qui signalent la dangerosité de cette piste.

- "Ce message peut être assez difficile à recevoir pour les femmes victimes de violence, parce qu'on parle beaucoup de communication puis on parle beaucoup de l'apport des deux parents dans l'éducation des enfants. Ce n'est pas adéquat. Pour moi, c'est une manière de rectitude politique » (intervenante en violence conjugale).

Les parents et les professionnels rencontrés adhèrent généralement au principe pacificateur de la médiation familiale et valorisent la négociation plutôt que le registre conflictuel caractéristique du Tribunal de la famille, mais leurs expériences se révèlent contrastées à cet égard. Dans certains cas, la médiation a aidé à identifier des solutions pratiques : "Ce que j'aimais avec "avoir une médiatrice", c'est [qu']elle était là, pas pour moi, pas pour mon ex, elle était là pour les enfants. Alors, elle nous a guidés, je pense, très bien là-dedans » (mère en garde partagée).

En revanche, dans les cas de ruptures hautement conflictuelles ou empreintes de violence conjugale, les intervenants suggèrent d'explorer $d^{\prime}$ 'autres pistes (Cyr et al., 2013). En l'absence $d^{\prime}$ une capacité minimale de communication ${ }^{(24)}$, la médiation ne semble pas recommandable. Certains médiateurs refusent même d'agir dans ces circonstances ou imposent alors des règles très strictes.

- "Moi, je n'ai pas de tolérance pour ça. S'il y a eu de la violence et/ou qu'il y a encore de la violence, je n'accepte pas de faire le dossier en médiation. Ou [uniquement] avec la nuance suivante : je vais [...] rencontrer la personne [...] victime de violence pour savoir comment elle se sent d'être dans la même pièce que la personne qui a été violente à son égard » (avocate-médiatrice).

En effet, I'efficacité de la médiation familiale, ou de toute justice collaborative, doit être questionnée si les parties concernées ne collaborent pas.

- "Ça s'est très mal passé je vous dirais. Parce qu'on n'arrivait pas à s'entendre sur les détails les plus insignifiants puis les plus élémentaires... À mon humble avis, ce n'est pas l'absence de communication qui rend la garde partagée difficile, mais l'absence de communication est un symptôme qu'en dessous, il y a des choses malsaines. Et ce sont ces choses malsaines qui rendront la garde partagée difficile » (père en garde partagée).

Or, la philosophie de la médiation familiale professionnelle au Québec est traversée par le refus de considérer l'inégalité des rapports sociaux de sexes au sein d'un couple et la violence postséparation. Plusieurs parents ne sont pas prêts à adopter cette nouvelle gestion contractuelle de la vie familiale proposée par les séances obligatoires. La garde partagée favoriserait le déni des statuts inégaux au sein d'un couple, en situation de violence tout particulièrement, ainsi qu'une polarisation entre ceux et celles qui peuvent assumer pleinement cette « autonomie

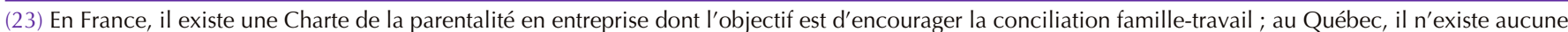

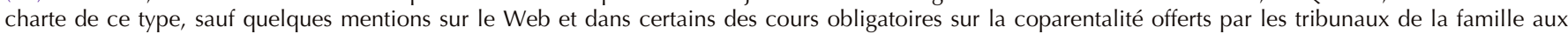
parents négociant la garde de leurs enfants.

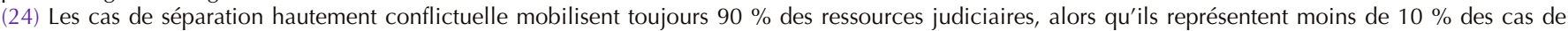
séparation enregistrés au tribunal.

Revue des politiques sociales et familiales $n^{\circ} 128-3^{\text {e }}$ trimestre 2018

42 Politique familiale 
individuelle », posée comme nouvel idéal, et ceux et celles dont la position n'est pas assurée ou qui ont besoin $\mathrm{d}^{\prime}$ attaches et de protection dans le cadre de leur vie familiale (Castel, 1995). Les inégalités au sein du couple hétérosexuel ne sont pas abordées au sein de ces séances obligatoires ni les aspects sociologiques de la transformation des rôles parentaux et des rapports sociaux de sexe. Les juges du tribunal de la famille sont rattachés à la Cour supérieure du Québec et accueillent aussi bien les litiges commerciaux que familiaux. Ils n'ont aucune formation précise en sociologie ou en psychologie (Tétrault, 2014) et véhiculent ainsi leur conception personnelle de l'idéal à atteindre en matière de garde parentale.

\section{De la garde partagée consentie à la garde partagée imposée}

Comment réagissent les parents à ces nouvelles normes favorisant la garde partagée ? Très peu d'écrits abordent cette question au Québec. Des recherches antérieures (Côté et Gaborean, 2015 ; Côté, 2000, 2006 et 2012) ainsi que les entretiens récents permettent néanmoins $d^{\prime}$ 'avancer certains éléments de réponse. Depuis plus de quinze ans, la garde partagée est prisée par des jeunes parents québécois séparés à double insertion professionnelle (Côté, 2000 et 2006). Ils la choisissent souvent pour exercer une parentalité plus horizontale et mieux répondre aux exigences de la conciliation famille-travail, car la taille des familles se rétrécit. Dans les cas très litigieux, la garde partagée est aussi parfois demandée par un parent avec l'idée de réduire la pension alimentaire ou pour exercer des pressions sur son exconjoint-e (Côté, 2006 et 2012). La popularité de la garde partagée ne diminue pas pour autant sa complexité logistique et relationnelle (Côté, 2000). Dans sa forme volontaire (négociée à l'amiable entre les deux parents), la garde partagée s'appuie sur une confiance croisée des parents dans leurs capacités respectives (Côté, 2004). Elle fait habituellement l'objet d'une entente cadre négociée, mais souvent informelle. Contrairement à ce qu'affirment Émilie Biland et Gabrielle Shütz (2013), les recherches montrent que la garde partagée reste accessible aux parents québécois disposant de revenus variables (Côté, 2006) ${ }^{(25)}$. Le modèle d'horizontalité et de partage de la charge parentale de la garde partagée est populaire auprès des couples à double mobilité professionnelle, lorsque les grands-mères sont insérées professionnellement et que la mère possède une forte identité professionnelle. Enfin, l'enjeu de la pension alimentaire est plus important pour les pères des catégories sociales aux revenus supérieurs, puisqu'ils seront sujets à une importante pension alimentaire.

L'organisation du temps de présence de l'enfant à chaque domicile parental ainsi que la responsabilité économique sont conçues sur le principe de partage symétrique (moitié-moitié). Des stratégies résidentielles, spatiales et temporelles complexes sont mises en œuvre par les parents pour effectuer ce partage symétrique du temps de garde. Le modèle de symétrie est incorporé par les parents qui ont recours à cette représentation parentale moitiémoitié comme garante de la symétrie des rôles et des droits. Cependant, ni le partage financier ni le partage de la charge éducative et des soins ne sont réellement symétriques : les mères assument plus de tâches et de responsabilités financières que les pères même si leurs revenus sont, en règle générale, plus faibles (Côté, 2000, 2004 et 2012). Ces usages parentaux en garde partagée révèlent encore aujourd'hui, au Québec, un écart entre la représentation de symétrie et les pratiques de partage.

- «Oui, c'est partagé, mais sauf qu'il ne me paie pas. Il ne m'a pas payé encore... Il me dit qu'il a de petits problèmes d'argent et il ne me paie pas... Ah ! II a fait des rénovations, il a payé un voyage à [ma fille] en Floride au mois de mars. Ça, il a de l'argent pour ça, pas pour l'essentiel [rire] » (mère en garde partagée).

- "En règle générale, les femmes en font un peu plus même si les hommes en font de plus en plus, puis ils s'impliquent auprès des enfants. Je ne crois pas, moi, au partage égal à cinquante-cinquante, ce n'est pas vrai » (mère en garde partagée).

Malgré la nature asymétrique du partage, la garde partagée semble permettre aux mères de diminuer la charge qu'elles assumaient lorsque qu'elles vivaient en couple. Le partage moitié-moitié du temps de garde les libère, en effet, de plusieurs responsabilités :

- "Je trouve ça plus facile maintenant que je suis séparée. Avant, c'était moi qui m'occupais de tout. Alors

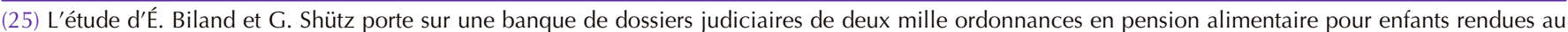

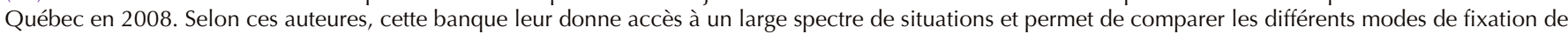

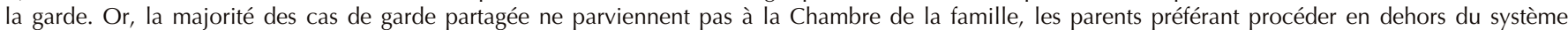

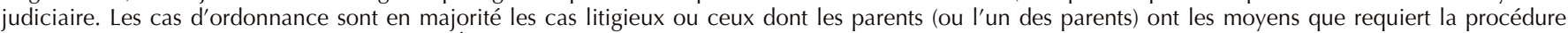
juridique. Pour ces raisons, les conclusions d'É. Biland et G. Shütz ne peuvent se généraliser à l'ensemble des parents québécois en garde partagée.

Revue des politiques sociales et familiales $\mathrm{n}^{\circ} 128-3^{\mathrm{e}}$ trimestre 2018

43 Politique familiale 
qu'aujourd'hui j'ai les enfants cinquante pour cent du temps, c'est cinquante pour cent moins de travail !... Donc, aujourd'hui, je me dis que ces aspects-là sont plus faciles à gérer pour moi » (mère en garde partagée).

Quant aux pères, ils assument en règle générale plus de tâches éducatives ou domestiques que lorsqu'ils étaient en couple. Plusieurs ont en effet appris à cuisiner ou à s'occuper des enfants, ils s'investissent dans une paternité différente depuis qu'ils sont en garde partagée :

- « En garde partagée, je peux assumer davantage mon rôle de parent, avoir une relation avec les enfants plus saine, dépourvue d'un objecteur moral, de quelqu'un qui me dit tout le temps quoi faire. Les liens sociaux se sont améliorés. J'ai plus de tâches, mais je le fais à mon rythme » (père en garde partagée).

Les modalités de garde adoptées par les parents sont souvent souples et se transforment parfois en fonction des besoins. En règle générale, elles adoptent la formule classique une semaine-une semaine (1/1). En revanche, les formules $2 / 5,5 / 2,3 / 4,4 / 3$ sont aussi très prisées. Le "nesting »(26) et la circulation libre entre deux maisons sont également utilisés par certains parents.

Dans les cas litigieux, les motifs d'octroi de la garde partagée invoqués par les juges sont les suivants : l'intérêt de l'enfant, le point de vue de l'enfant lorsque celui-ci a l'âge requis, le point de vue des experts ainsi que les capacités parentales (Québec, 2002, p. 57). L'opinion des parents y est curieusement absente : selon des parents en litige interrogés, les juges les consultent peu :

- "Je suis là, je dis oui, je réponds au juge. Et il ne me questionne pas, il ne parle pas avec moi. Comment fait-il pour prendre ces décisions-là? C'est quelle lentille qu'il prend ? Je ne parle pas » (mère en garde partagée).

- " [Le juge a accordé] la garde partagée. Comme ça. Sans lire les affaires. Il ne m'a même pas écoutée on dirait : "Ah! Bien, je rends mon verdict", puis c'est tout !, wow! » (mère en garde partagée).

Ces parents considèrent que la communication entre les ex-conjoints devrait avoir la préséance pour la mise en place d'une garde partagée. La pression psychologique exercée par l'un des conjoints, l'absence de confiance mutuelle et une communication déficiente ou encore la violence rendraient la garde partagée impraticable, selon ces parents. Car l'enfant risque d'être la victime des règlements de compte :

- "Je ne pourrais pas accepter qu'on m'impose une garde partagée quand on ne prend pas en considération le contexte communicationnel que j'ai avec le père. Le juge doit voir au bien-être de l'enfant, mais pour que le bien-être de l'enfant se fasse, il faut aussi qu'il observe de quelle manière les deux parents agissent ensemble. Quelles répercussions ou quel impact ça va avoir sur l'enfant " (mère en garde partagée).

Ces conditions minimales dressées par les parents ne sont habituellement pas prises en compte dans I'arène judiciaire. Une étude des jugements récents réalisée par Michel Tétrault (2014) indique que le critère de communication entre les ex-conjoints pèse de moins en moins dans les jugements de garde : de l'avis de plusieurs juges, la garde partagée pourrait même fonctionner lorsque la communication entre les parents est défaillante (Tétrault, 2014, p. 17). Imposée par le tribunal ou négociée entre les parents, la garde partagée est ainsi devenue au Québec une norme sociale : plusieurs parents ont même adopté l'idée que cette forme de garde permet de mieux respecter l'intérêt de l'enfant. Les pressions sociales favorables à la garde partagée ont une incidence sur leurs décisions. Elle est « dans l'air du temps » et correspond aux idées progressistes :

- «On se croit des gens libéraux ou des progressistes. Toutes les idées nouvelles qu'on voyait sur Internet, on les adoptait. Alors, la garde partagée, c'était comme donné, parce qu'on savait que c'était mieux pour les enfants. Puis je savais que la garde partagée serait toujours privilégiée par un juge »(père en garde partagée).

- "Dans ma tête, il était clair qu'il n'y avait aucune autre décision possible que la garde partagée. Moi, j'avais envie d'être père de famille, puis de l'être comme du monde » (père en garde partagée).

- "Dans ma tête, tout le monde qui se sépare a la garde partagée, sauf s'il y a quelque chose d'extrêmement grave... c'est social, tous les parents qui sont séparés ont la garde partagée » (mère en garde partagée).

\section{Conclusion}

La préférence de facto pour la garde physique partagée au Québec a pu être mise en place grâce à l'action simultanée des systèmes législatif (obligation de suivre les cours de

(26) «Partage de la résidence familiale ». Les enfants ne changent pas de domicile, ce sont les parents qui vont habiter avec les enfants à tour de rôle.

Revue des politiques sociales et familiales $\mid n^{\circ} 128-3^{\text {e }}$ trimestre 2018

44 Politique familiale 
coparentalité et sur la médiation familiale), administratif (gratuité des sessions de médiation, dispositifs de pensions alimentaires, dispositifs fiscaux) et professionnel (médiateurs, travailleurs sociaux, experts du champ de la famille). Le dispositif de médiation familiale est devenu l'une des assises centrales de cette mise en place de la garde partagée. Plusieurs politiques administratives ont été modifiées à la lumière du principe de partage symétrique du temps de garde parentale. Elles ont servi de fondement à la construction de la nouvelle norme de partage symétrique des rôles parentaux et, par la même occasion, de la garde physique partagée comme idéal d'égalité et de démocratie postséparation. Y ont aussi contribué les nouvelles théories psychologiques ainsi que l'avis de professionnels selon lesquels la garde partagée correspondrait à l'intérêt supérieur de l'enfant tel que codifié dans les conventions internationales (Millar, 2009).

Adoptée par plusieurs parents sur une base volontaire, mais toujours moins répandue que la garde maternelle, la garde partagée a résonné, dans l'imaginaire collectif québécois, grâce aux valeurs d'égalité entre les mères et les pères qu'elle a projetées. Cependant, la promotion par les dispositifs administratifs, professionnels et juridiques I'a transformée en norme. Ainsi, la garde maternelle est désormais accordée plus rarement par la Chambre de la famille lorsque les parents ne s'accordent pas. Car plusieurs juges et professionnels sont maintenant convaincus, à tort, que la garde physique partagée pourrait même remédier aux conflits parentaux ou à la violence conjugale. Trop de professionnels du domaine familial considèrent aussi que la garde partagée mettrait fin aux inégalités entre les pères et les mères séparés. Or, les entretiens ont révélé, au contraire, que cette nouvelle norme est rejetée, souvent en sourdine, par les parents et les professionnels parce qu'elle ne correspond pas nécessairement aux désirs et aux intérêts des pères et des mères séparés, ni à ceux de tous les enfants concernés.

La pression à la conformité au nom d'une symétrie et d'une indistinction des rôles parentaux (Jamoulle, 2005) aura imposé au Québec cette redéfinition des rapports sociaux de sexe en matière de garde parentale postséparation. Celle-ci se sera effectuée sans débat public ni assise scientifique, les parties prenantes agissant chacune en silo à la lumière de conceptions émergentes sur la symétrie parentale (Côté, 2004), créant, par la même occasion, de nouveaux effets sexués. Les données empiriques sur la garde physique partagée confirment ainsi la thèse de B. Bastard (2006) selon laquelle de nouvelles formes de régulation familiale, véhiculées et promues par des intervenants du champ psychosocial et centrées sur le maintien des liens parentaux après rupture, sont soumises à des exigences paradoxales. Elles renforcent le contrôle en matière de garde sur le couple parental tout en prônant la diversité et l'autonomie de leur fonctionnement.

- Bastard B., 2012, Médiation familiale : une profession qui peine à trouver son public, Informations sociales, vol. $2, \mathrm{n}^{\circ} 170$, p. 66-73.

- Bastard B., 2006, Une nouvelle police de la parentalité ?, Enfances, Familles, Générations, n 5, p. 11-21.

- Belleau M.-C., Talbot-Lachance G., 2008, La valeur juridique des ententes issues de la médiation familiale : présentation des mésententes doctrinales et jurisprudentielles, Les Cahiers de droit, vol. 49, $\mathrm{n}^{\circ} 4$, décembre, p. 607-653.

- Biland É., Shütz G., 2013, La garde des enfants de parents séparés au Québec. Une analyse quantitative des dossiers judiciaires, collection Que savons-nous, Québec, Aruc- Séparation parentale, recomposition familiale.

- Canada, Agence du revenu du Canada, 2017, Allocation canadienne pour enfants et les programmes provinciaux et territoriaux connexes. Pour la période de juillet 2017 à juin 2018. Ottawa, éditeur officiel.

- Canada, ministère de la Justice, 2000, Réforme du divorce et exercice conjoint de l'autorité parentale. La perspective du droit civil québécois, Document de référence préparé et présenté par Goubau D., Ottawa, éditeur officiel.

- Canada, ministère de la Justice, 1997, Lignes directrices fédérales sur les pensions alimentaires pour enfants.

- Canada, Comité mixte spécial sur la garde et le droit de visite des enfants, 1999, Pour l'amour des enfants, Ottawa, Parlement du Canada.

Revue des politiques sociales et familiales $n^{\circ} 128$ - $3^{\text {e }}$ trimestre 2018

45 Politique familiale 
- Castel R., 1995, Les métamorphoses de la question sociale. Une chronique du salariat, Paris, Fayard.

- Clairmont S., Timmermans H. et al., 2014, Parentalité après la rupture, Couple un jour... parents toujours, Montréal, Gouvernement du Québec.

- Collombet C., 2014, Consensus et médiation dans les séparations parentales en Europe, Politiques sociales et familiales, $\mathrm{n}^{\circ} 117$, p. 75-79.

- Commaille, J., 2006, L'économie sociopolitique des liens familiaux, Dialogue, vol. 174, n 4, p. 95-105.

- Côté D., 2012, "Mais je voulais que ça cesse! » : récits de mères sur la garde partagée et la violence postséparation, Nouvelles pratiques sociales, vol. 25, n 1, p. 44-61.

- Côté D., 2006, D'une pratique contre-culturelle à l'idéal-type : la garde partagée comme phénomène social, Revue québécoise de psychologie, vol. 27, n 1, p. 15-43.

- Côté D., 2004, La garde partagée des enfants : nouvelles solidarités parentales ou renouveau patriarcal ?, Nouvelles questions féministes, vol. 23, n 3, p. 80-95.

- Côté D., 2000, La garde partagée, l'équité en question, Montréal, Remue-ménage.

- Côté D., Gaborean F., 2015. Nouvelles normativités de la famille : la garde partagée au Québec, en France et en Belgique, Revue canadienne Femmes et droit, vol. 27, n 1, p. 22-46.

- Cyr F., 2008, Débat sur la garde partagée : vers une position plus nuancée dans le meilleur intérêt de l'enfant, Santé mentale au Québec, vol. 33, n 1, printemps, p. 235-251.

- Cyr, F., 2006, La recherche peut-elle éclairer nos pratiques et aider à mettre un terme à la polémique concernant la garde partagée? Revue québécoise de psychologie, vol. 27, n 1, p. 79-114.

- Cyr F., Di Stefano G., Desjardins B., 2013, Family life, parental separation, and child custody in Canada : a focus on Québec, Family Court Review, vol. 51, n 4, p. 522-541.

- Cyr F., Lavoie É., Chagnon M., 2011, Prévalence de la garde partagée chez les familles québécoises ayant un enfant né en 1997-1998 : profil sociodémographique et psychologique. rapport de recherche, ministère de la Justice du Québec.

- Desrosiers H., Simard M., 2010, Diversité et mouvance familiales durant la petite enfance, Institut de la statistique du Québec, L'ÉLDEQ 1998-2010 en bref, vol. 4, fascicule 4.

- Gagnon M., 2006, Les mythes de la garde partagée, Revue québécoise de psychologie, vol. 27, n 1, p. 47-78.

- Gauthier Y., 2008, Les enfants sont-ils les cobayes de la présomption du tribunal en faveur de la garde partagée?, Santé mentale au Québec, vol. 33, nº 1, p. 203-208.

- Giddens A., 2004, La transformation de l'intimité, Paris, Chambon.

- Goubau D., 2009, L'évolution de la place de l'enfant au sein du droit de la famille séparée au Canada, in Fédération des associations de juristes d'expression française (Fajef) et Association internationale francophone des intervenants auprès des familles séparées (Aifi), Du droit de l'enfant à l'intérêt de la famille séparée, Cowansville, Éditions Yvon Blais, p.1-20.

- Guillemard S., 2012, Médiation, justice et droit : un mélange hétéroclite, Les Cahiers du Droit, vol. 53, n², juin, p. 189-228.

- Hachet B., 2014, Les calendriers et les agendas de la résidence alternée. Structure et plasticité des territoires temporels des parents, Politiques sociales et familiales, $n^{\circ} 117$, p. 29-44.

- Jamoulle P., 2005, Des hommes sur le fil. La construction de l'identité masculine en milieu précaire, Paris, La Découverte.

- Joyal R., 2004, L'attribution de garde des enfants après le divorce ou la séparation des parents : Perceptions d'avocates et d'avocats en droit de la famille, Revue du Barreau, vol. 64, n 2, p. 445-453. 
- Joyal R., 2003, Garde partagée de l'enfant - Constat et réflexions à la lumière de recherches récentes, Cahiers de droit, vol. 44, n² 2, p. 267-280.

- Juby H., Le Bourdais C., Marcil-Gratton N., 2005, Sharing roles, sharing custody ? Couples' characteristics and children's living arrangements at separation, Journal of Marriage and Family, n 67, p. 157-172.

- Juston M., Gargoullaud S., 2014, Médiation familiale et contrats de coparentalité, ministère des Affaires sociales et de la Santé, Paris, La Documentation française.

- Le Roy I., 2006, La garde partagée...une présomption jurisprudentielle?, Revue québécoise de psychologie, vol. 27, $\mathrm{n}^{\circ} 1$, p. 33-46.

- Millar P., 2009. The best interests of children, Toronto, University of Toronto Press.

- Neyrand G., 2014, La résidence alternée ou le défi de la coparentalité, Politiques sociales et familiales, $\mathrm{n}^{\circ} 117$, p. 5-15.

- Ouellet J., 2012, Adaptation de l'enfant, conflits parentaux et relations parents-enfant en garde partagée et en garde seule, thèse de doctorat en psychologie sous la direction de Sylvie Drapeau, Faculté des sciences sociales, Université Laval, Québec.

- Québec, ministère de la Justice, 2014 a, Le modèle québécois de fixation des pensions alimentaires pour enfants, Brochure d'information à l'intention des parents séparés, Québec, Éditeur officiel.

- Québec, Régime des rentes de Québec, 2014 b, Vous partagez les conditions de garde, page web d'information à destination des parents séparés, http://www.rrq.gouv.qc.ca/fr/enfants/changement_garde/Pages/obtenez_ garde_partagee.aspx.

- Québec, ministère de la Justice, 2013, Code de procédure civil, Publications du Québec, section 5, articles 814.3 à 814.14.

- Québec, ministère de la Justice, direction des orientations et politiques aux affaires familiales, 2012 a, Séance sur la parentalité après rupture, Rapport d'évaluation, Québec, Éditeur officiel.

- Québec, ministère de la Justice, 2012 b, Rapport d'évaluation - Séance sur la parentalité après rupture (projet pilote), Québec, Éditeur officiel.

- Québec, ministère de la Culture, des Communications et de la Condition féminine, 2011 a, Plan d'action gouvernemental pour l'égalité entre les hommes et les femmes, Québec, Éditeur officiel.

- Québec, ministère de la Famille et des Aînés, 2011 b, Bilan 2006-2011 des réalisations en faveur des familles et des enfants, Québec, Éditeur officiel.

- Québec, Institut de la statistique du Québec, 2011 c, Bilan démographique du Québec, Québec, Éditeur officiel.

- Québec, Bureau de normalisation, 2010, Conciliation travail-famille, Norme BNQ 9700-820.

- Québec, ministère de l'Emploi, de la Solidarité sociale et de la Famille, 2002 (rédigé par R. Joyal, Laperrière-Adamcyk É., Le Bourdais, C. et Marcil-Gratton N.), Le rôle des tribunaux dans la prise en charge des enfants après la séparation ou le divorce, Québec, Éditeur officiel.

- Québec, 1997, Loi instituant au Code de procédure civile la médiation préalable en matière familiale et modifiant d'autres dispositions de ce code, Gazette officielle du Québec, $129^{e}$ année, nº 29.

- Sassier M., 2001, Construire la médiation familiale. Paris, Dunod.

- Tétrault M., 2014, La garde exclusive : une exclusivité... Ou comment on crée une présomption de garde partagée, Montréal, Yvon Blais.

- Wynants B., Willeman N., Guislain C. et Marquet J., 2009, Comment favoriser le recours à la médiation familiale dans les conflits familiaux ?, Rapport de recherche pour le Secrétaire d'État à la Politique des familles, Université catholique de Louvain, Centre interdisciplinaire de recherche sur les familles et les sexualités (Cirfase). 